\title{
Conflits de générations et célébrations nationales : analyse et perspectives
}

Présentation de Bernard LACROIX

\section{Bernard Lacroix}

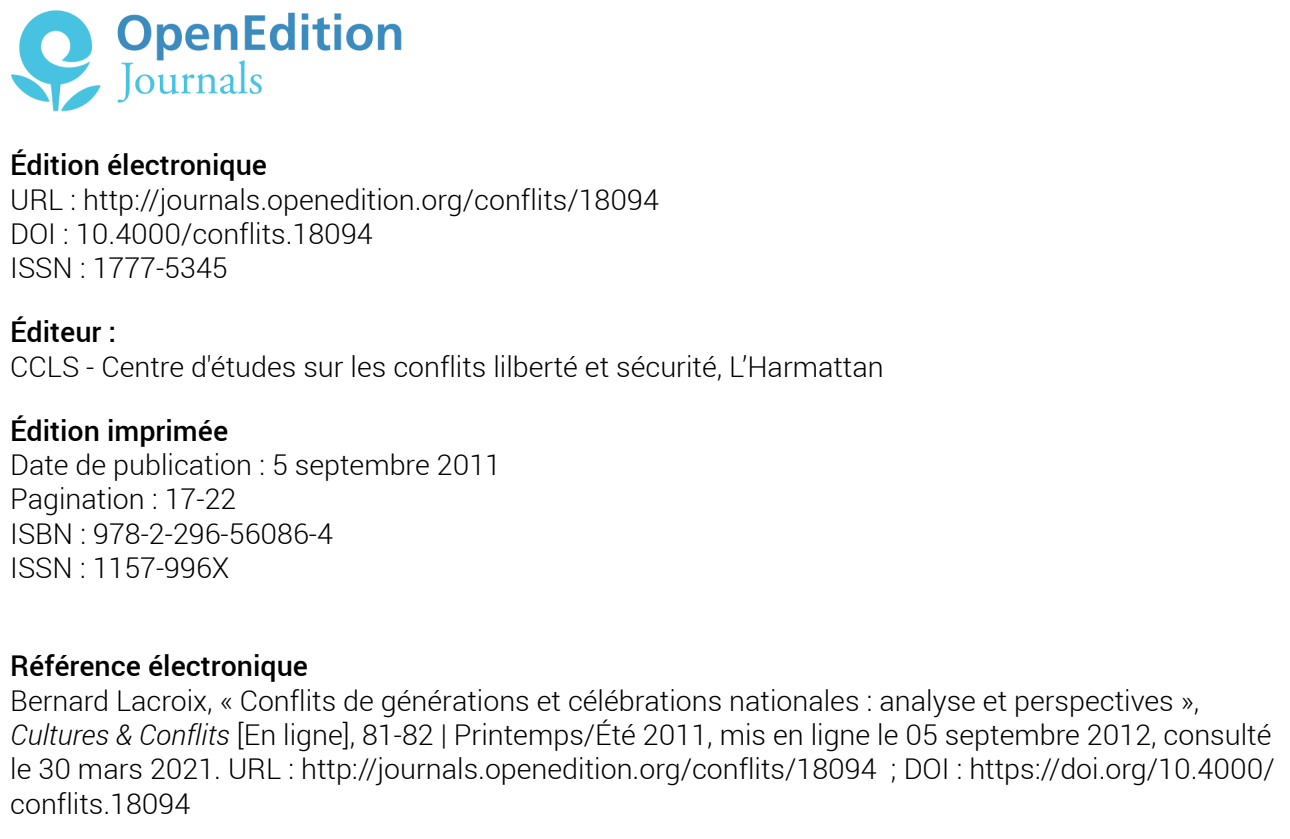

Creative Commons License 


\section{Conflits de générations et célébrations nationales : analyse et perspectives}

\section{Présentation de Bernard LACROIX}

Bernard Lacroix est Professeur de science politique à l'Université Paris Onest Nanterre et Membre du Comité de Rédaction de Cultures \& Conflits.

\section{$\mathrm{P}$} ourquoi introduire un ensemble de contributions relatives à la violence en politique par quelques pages de Norbert Elias empruntées à ses réflexions sur l'Allemagne ? Le texte est inédit en français, et cela pourrait être une raison suffisante. Il y a de quoi s'inquiéter aujourd'hui de la légèreté de trop d'éditeurs français, satisfaits d'une diffusion limitée d'un des sociologues majeurs du siècle passé, quand Allemands et Anglais parviennent à faire connaître, dans son intégralité, le cheminement et l'étendue d'un travail exemplaire. Mais ce travail est surtout la mise à plat d'un formidable paradoxe intellectuel : le spectacle de l'exacerbation d'une forme paroxystique de violence dans l'une des sociétés apparemment les plus apaisées d'après-guerre. Les « années de plomb », si elles ne s’y résument pas, évoquent immédiatement le nom de la Fraction Armée Rouge, plus connue sous le patronyme de son chef, comme « la bande à Baader », responsable entre 1968 et 1993 d'un peu plus d'une trentaine d'assassinats et de nombreux attentats. L'effroi qui pousse à l'indignation devant cette violence assumée et revendiquée ne permet cependant d'en comprendre ni l'avènement ni la nécessité.

Les pages qui suivent proviennent des Considérations sur l'Allemagne (Über die Deutschen) et plus exactement de sa partie centrale : "Rapports entre violence et civilisation : remarque sur le monopole de la violence physique et ses fractures 1 ». Le livre, le dernier ouvrage publié par Elias de son

1. Elias N. «Studien über die Deutschen. Machtkämpfe une Habitusentwicklung im 19. und 20. Jahrhundert », in Gesammelteschriften, volume 11, pp. 358-393, Francfort, Suhrkamp, 2005, et pour l'édition de poche, Studien über die Deutschen. Machtkämpfe une Habitusentwicklung im 19. und 20. Jabrhundert, Francfort, Suhrkamp STW, 1992, pp. 318348. Pour une traduction anglaise, le lecteur pourra aussi se reporter à The Germans: Power Struggles and the Development of Habitus in the Nineteenth and Twentieth Centuries, Cambridge, Polity Press, 1996, avec une préface de Eric Dunning et Stephen Mennell. En 2013, sous le titre Studies on the Germans, ce texte composera le volume 11 d'une traduction raisonnée de l'œuvre d'Elias entreprise par l’Université de Dublin. 
vivant, est paru en 1989. Il résulte d'une compilation de travaux passés et parfois anciens, de compléments, de coupures, de publications de circonstance ou de reformulations et il est le fruit d'une étroite collaboration avec Michaël Schroeter ${ }^{2}$. C'est cet ensemble d'écrits, initialement rassemblés par un traducteur progressivement devenu collaborateur, qui est devenu le texte de référence de l'édition allemande des œuvres complètes ${ }^{3}$. Ceci se fit à partir de l'inclination de ce dernier, parfois avec l'assentiment complet de l'auteur, parfois à son insu, dans un climat de difficultés croissantes avec « le grand homme » mais le plus souvent dans une atmosphère de déférence, de confiance et au total de révérence.

Pour ce qui concerne l'extrait traduit, Schroeter laisse entendre que celuici provient de liasses conservées par Elias dans ses multiples déplacements antérieurs (est-ce la fameuse malle transportée dans ses bagages qu'évoquent toujours allusivement et mystérieusement ceux qui ont travaillé avec lui ?) et que les observations, les documents ou les fragments rédigés ${ }^{4}$ ont servi à la préparation de l'exposé du 20e Congrès annuel des sociologues de langue allemande (Soziologentag) en septembre 1980, publié depuis dans les actes du colloque.

La référence à ce colloque n'est pas un point de repère anodin, pourvu qu'on n'entende pas en faire un accomplissement du travail d'Elias, ou bien le point de départ de recherches nouvelles qui aboutiraient au livre de 1989. Ce congrès, tenu à Brême, évoque inévitablement pour le vieux professeur ses premières armes universitaires devant des collègues, et le congrès de Zurich de 1928 qui avait vu l'affrontement à fleuret moucheté entre un fringant Karl Mannheim et un Alfred Weber crispé ; l'affrontement entre la volonté théorique de lier les conditions d'existence et les conceptions de la vie (ou ce qui revient au même, le refus de les dissocier) et un universalisme des valeurs de principe inconscient d'un libéralisme qui allait de soi ${ }^{5}$. En 1980, Elias a 83 ans. Il est enfin reconnu parmi les siens depuis la republication du Procès de civilisation, dans sa langue originale, en allemand, dix ans auparavant. Et il connaît une deuxième jeunesse scientifique avec le succès de l'ouvrage dans le monde universitaire et par delà les frontières, en France notamment, couronnée par la remise du prix Adorno en 1977. On peut se représenter, à l'occasion de ces journées de Brême, une discrète satisfaction d'être appelé à s'exprimer devant ses pairs et d'avoir recouvré le rang académique qu'Elias savait lui être dû, en dépit de la réserve qu'une pudeur blessée fait parfois passer pour de l'arrogance.

2. Schroeter M., Ehrfahrungen mit Norbert Elias, Francfort, Suhrkamp, STW, 1997.

3. Voir notamment la présentation de Nico Wilterdink in Elias N., Gesammelteschriften, op. cit., pp. 637-639.

4. Schroeter M., Erfahrungen..., op. cit., pp. 311-318 et spécialement pour l'extrait traduit ici, p. 315.

5. Voir Elias N., Norbert Elias par lui-même, Paris, Fayard, 1991, pp. 136-149, ainsi que, sur l'univers intellectuel des protagonistes de la sociologie allemande autour de Heidelberg, l'ou- 
Quant à Schroeter, il avait 32 ans lorsqu'il rencontra Elias en 1976 : « mes études derrière moi et tendance à les fuir... Mais ma soif d'identification était loin d'être assouvie... Ce n'est guère que dans les livres, ceux de Freud en particulier, que je trouvais un maître au sens le plus fort du terme... Mais au delà de toute inclination personnelle, c'était le destin d' un groupe en son entier que cachait cette réaction. Je ne sais si génération d'étudiants, ailleurs qu’à Berlin, fut plus étrangère à ses maîtres et à leurs enseignements... C'étaient davantage nos lectures personnelles et nos cercles enflammés qui étaient nos lieux de nourriture intellectuelle plutôt que les séminaires de nos professeurs 6 ».

Le retour sur des liasses jaunies du passé et sur les documents qui ont servi à préparer l'intervention formelle du Soziologentag tire donc aussi son sens d'échanges réguliers et répétés avec Schroeter qui baignent dans les circonstances politiques et académiques dont l'intervention de 1980 et le livre ultérieur sont autant d'expressions officielles. Nous avons, pour ce qui nous concerne, une image très floue de la plupart des points de fuite qui retiennent l'attention, de l'autre côté du Rhin. Et nous ne sommes pas de plain-pied avec dates, manifestations ou événements, et pas non plus avec les confrontations qu'elles ont engendrées entre l'Elbe et l'Oder, Bavière et Nordsee. Par exemple, avec ce que certains se donnent la facilité d'appeler un « mai 68 allemand » qui cristallise en... 1967 avec la venue du shah d'Iran, le problème du désarmement et la marche de Pâques, puis des protestations collectives suscitées par la mort de Benno Ohnesorg, un étudiant tué par la police lors d'une manifestation. Avec une réconciliation achevée en 1966 sous le patronage d'une «grande coalition » entre sociaux-démocrates et conservateurs, engluée dans l'impuissance de la bienséance parlementaire. Avec une poussée de critique étudiante pétrie de vision marxiste qui démasque dans la presse Springer, la main de l'attentat contre Rudi Dutschke ${ }^{7}$. Avec l'assèchement de cette opposition extra-institutionnelle, et sa métamorphose en groupes clandestins qui n’apparaissent publiquement qu'ultérieurement.

Décapitée, elle renaît aussitôt. Emprisonnée, elle se régénère sous les traits d'une génération nouvelle. Elle engendre bientôt la stupéfiante escalade de la seconde moitié des années 1970 justement : depuis l'enlèvement de Peter Lorenz, tête de liste CDU à Berlin, jusqu'à la prise d'otage de Stockholm, depuis la lutte récurrente sur les conditions de détention à la prison de Stammheim, jusqu'aux assassinats - celui du procureur général Buback, de Jürgen Ponto, directeur de la Dresdner Bank puis celui du président du patro-

vrage indispensable de Blomert R., Intellektuelle im Aufbruch Karl Mannheim, Alfred Weber, Norbert Elias und die Heidelberger Sozialwissenschaften der Zwischenkriegszeit, Carl Hanser Verlag, Munich, 1999.

6. Schroeter M., Erfahrungen..., op. cit., p. 270.

7. On pourra sur ces années se reporter à Gilcher-Holtey I., «Éléments pour une histoire comparée de Mai 68 en France et en Allemagne ", Scalpel, Cabiers de sociologie politique de Nanterre, 1999, 4-5, pp. 187-193. 
nat allemand, Hans Martin Schleyer en 1977 - qui complètent la panoplie des moyens d'intervention comme le détournement d'avion, pour authentifier et appuyer des revendications.

On peut faire ainsi un peu plus qu'une hypothèse et imaginer trouver dans la recontextualisation de la coopération entre le vieil homme et le jeune « assistant » quelques raisons à l'énigme du caractère ressassé d'une réflexion ruminée et de reprises récurrentes qui sont devenues un livre : comment et pourquoi, au delà de la dimension d'exception - par son volume, sa durée et ses conséquences en terme de répression - la lutte armée des années 1970-90, intéresse-t-elle autant un sociologue que la longue expérience de l'exil a éloigné de l'Allemagne ? Pourquoi, en particulier, « l'historien » spécialiste de la genèse des formes de comportement collectif reconnues et fortifiées par la présence de l'État, trouve-t-il dans une forme de violence contre des personnes supposées incarner l'État, mais également dans la mise en question radicale de l'outil d'exception de réalisations collectives - de promotions et de démultiplication -, la raison existentielle d'une obsession récurrente ? Enfin et surtout n'est-ce pas l'aspect infiniment singulier de l'État, qui revient sur le tapis ? L'aspect infiniment singulier, dès qu'on échappe à la figure d'une obsession collective illusoire qui n'existe pas de la même façon pour tous, d'un ensemble de relations sociales consolidées ? L'aspect infiniment singulier d'un ensemble de relations sociales qui s'objectivent en particulier dans leur reproduction ? L'aspect infiniment singulier d'un ensemble de relations qui n'existent qu'à travers la transcendance dont elles s'acquittent, qui s'imposent au lecteur, comme cette obsession et cette transcendance s'est imposée aux deux auteurs ? Chacune de ces formulations n'est qu'une façon de remettre sur le métier la question de la possibilité et de la légitimité d'une violence contre l'État, pour un homme qui a voué son travail et une partie de son existence, à montrer que « l'État » pouvait devenir, dans certaines circonstances et dans un espace circonscrit, l'instrument d'une forme de « civilisation ».

La suite se déduit aisément de cette crispation intérieure. L'assistant éditeur de formation littéraire, fait ressortir combien à ses yeux, une suite de fragments successifs, une mosaïque de pièces et de morceaux, coupée de ruptures de rythme et de ton, sont étrangères au « beau style » que cultive l'amour de la langue. Et de fait, le texte, comme certains pourront en avoir le sentiment au fil d'une première lecture, étale à l'envi ses reprises et ses répétitions, chemine par des détours qui ne paraissent pas s'imposer, quand il ne fait pas la place à des compléments donnant l'impression d'être étrangers au développement. Mais il y a plus accablant. Rien ici qui semble satisfaire un quelconque impératif du travail empirique au sens ordinaire du terme, rien qui ressemble à un quelconque recueil de données, ou à une exégèse de tableaux qui mène aux quelques facteurs de l'équation exemplaire plaisante pour l'esprit. Mais rien non plus qui ne détaille la révélation que délivrerait on ne sait quelle notion cachée, violence ou conflit de génération. Aucun empirisme en somme, façon 
Robert K. Merton années 1950, pour parler vite. Mais pas non plus le moindre théoréticisme, quelque chose comme le développement d'une grande idée à la Leo Strauss dont l'histoire serait l'accomplissement. Une succession de réflexions et de constats, tout au plus. Et comment ne pas se rendre à une forme d'évidence politique ? Les raisons d'être de la violence allemande des années 1970 ne sont pas dans l'analyse de la notion de violence. Et les conflits de génération, on l'oublie trop souvent, ont d'autres causes que celles auxquelles les réduisent ceux qui les vivent. Il faut peut être se dire alors que la voie étroite entre deux « obstacles épistémologiques » majeurs n’est pas si différente de ce qu'on appelait encore, dans les années 1970, rationalisme appliqué. A ce détail près que le vieux monsieur ne se sent pas tenu de reprendre en détail tout ce qui fait le fond commun de la sociologie : les effets politiques des bouleversements dans les rythmes d'ascension sociale ${ }^{8}$, les conséquences pratiques des transformations morphologiques, celles en particulier qui ne sont pas voulues et encore moins anticipées, ou l'effet de cliquet né de l'apparition de cette nouvelle forme de société qui s'incarne dans « l'État des partis », comme on peut le noter dès les premières pages de l'extrait proposé. Le texte devient ainsi exemplaire d'une «manière de faire » propre à l'auteur : déplier un schème explicatif pour comprendre des enchaînements que l'éparpillement apparent des faits et l'étirement de la chronologie rendent au premier abord inintelligibles. La dynamique des possibilités offertes et des fermetures d'opportunités pensée à travers des contextes différents de socialisation des groupes, balaye les hypothèses toutes faites (l'épuisement des régimes, le conflit transhistorique des générations, etc.) et permet de comprendre aussi bien la construction du "déclin » de la république de Weimar que les usages collectifs du passé hitlérien dans la période de l'après-seconde guerre.

Beaucoup souhaiteraient finalement faire de Norbert Elias un auteur fréquentable, en oubliant sa conviction inébranlable dans les vertus de la sociologie, soit qu'il faille renvoyer au passé un auteur qui connut naguère son heure de gloire, soit qu'ils consentent à le faire figurer dans le Panthéon des grands auteurs de l'histoire des idées. On aura pourtant ici (chemin faisant) une bonne illustration de la façon dont les réflexions d'Elias sont intrinsèquement une réflexion sur le pouvoir, en fait sur les relations de pouvoir qui ne peut laisser indifférentes des sciences sociales inquiètes du monde où elles se déploient, et des sciences politiques au sens large, entendues autrement que comme un conseil expert aux hommes politiques. Une réflexion rare et une réflexion $\mathrm{du}$

8. On regrettera évidemment le tour essentiellement et exclusivement «politique » emprunté par le « débat » sur « l’ascenseur social », et la réduction publique de ce débat à une discussion étroitement objectiviste autour du déclassement. On regrettera également l'ignorance surprenante, mais pas excessivement étonnante, du travail de politistes des années 1980, animés par la conviction qu'il n'est pas de science politique qui tienne sans interrogation préalable des mécanismes sociaux dont « la politique » est l'expression travestie - et par exemple du colloque des années 80 relatif aux classes moyennes animé par Georges Lavau publié sous le titre L'univers politique des classes moyennes (Paris, Presses de la FNSP, 1983) ainsi que quelques unes des discussions, le plus souvent ignorées, de Bernard Lacroix dans L'utopie communautaire (Paris, PUF, 1981, édition rééditée et augmentée en 2006). 
temps présent. Le drame à courte vue du travail intellectuel est trop souvent de confondre une dimension analytique du travail (par exemple le conflit de génération) avec une perspective d'explication dans son ensemble, en transformant insensiblement l'une en l'autre, ou à plus forte raison avec une perspective d'explication qui permette d'en tirer politiquement les conséquences qui s'imposent. Ou pour être plus clair encore, avec la définition des actions qui pourraient être tout autre chose que l'une ou l'autre des rationalisations sommaires d'apparence savante dont les professionnels de la politique et les apprentis professionnels de la politique se font les illusionnistes, en général contre d'autres professionnels de la politique, sans que les uns ni les autres ne sachent où cette confrontation les entraîne. Mais on éprouvera, du même coup, de quelle façon cette inquiétude théorique et pratique pour le pouvoir, ses ressorts et ses dynamiques n'est qu'une des faces de la réflexion du sociologue allemand en la matière. L'autre face de l'investigation est l'enquête sur les mécanismes de censure que supporte dorénavant l'existence de l'État. Il fait assez peu de doute que l'apparition de l'État comme phénomène social et les luttes autour de l'appropriation et de l'usage de l'État, entretiennent, selon les rapports de force et selon les circonstances, des rapports avec l'avenir des formes de tolérance et les modes de coexistence entre individus : on peut, à la suite de l'auteur, les appeler « la civilisation ». Mais il n'y a aucun lien nécessaire, encore moins de lien de continuité, entre l'apparition de l'État et l'une ou l'autre des formes routinières qu'il finit par emprunter, entre les " progrès » en matière de coexistence et de "civilité » et cette orientation d'ensemble. Ce second aspect, contrepartie nécessaire du premier, le retour de Norbert Elias sur les formes de censure qui naissent de l'existence de l'État, rend son tranchant à un "classique » menacé d'embaumement : en réhabilitant les raisons des recherches intellectuelles et les revendications armées par un travail d'intellectuels exclusivement mobilisés par ce type de travail, en un moment paradoxal d'asservissement planétaire de leur activité. Ou pour le dire d'un mot : en alimentant notre réflexion mais, en invitant aussi inévitablement, dans un moment de contre-révolution généralisée, à une forme de sursaut. 